\title{
Estimation Algorithm of Two-Dimensional Direction of Arrival based on Rectangle array of Distributed Source
}

\author{
Hui Yao, Ying Wu \\ Zhenzhou Information Science and Technology Institute \\ Zhenzhou, 450002, China \\ E-mail: hui56_y@126.com
}

\begin{abstract}
For estimating two-dimensional direction of arrival (DOA) of local scattering distributed source, the computational complexity of various algorithms for DOA is prohibitively high, induced by high dimension searching manipulation. According to this problem, an ESPRIT-like algorithm is proposed. The proposed algorithm exploits the rotational invariance properties of signal space, the DOA can be obtained without any peak-finding searching, which has significantly reduced the computational complexity. Simulations clearly demonstrate that the algorithm enjoys improved precision, and is more suitable for complicated environment.
\end{abstract}

Keywords-Coherently Distributed source; Azimuth angle; Elevation angle; ESPRIT; Rectangle array

\section{INTRODUCTION}

In recent years, high-precision array signal processing technology has been strongly paid attention to and is widely applied in the field of radar, sonar and wireless communication. Existing array signal processing methods are usually based on the assumption of point source. However, actual targets have more complex space distribution characteristics than point targets and signal dispersion phenomenon usually results in expansion of spatial domain, time domain and frequency domain. Point source is a logical mathematics approximation, which will reduce the complexity of relevant algorithm and analysis. Nevertheless, point source model often fails to describe characteristics of observed data of array while the space distribution characteristics of sources could not be ignored, which will lead to performance deterioration of highpowered parameters estimation algorithms. As a result, distributed sources model and interrelated parameters estimation technologies have a great deal of production both inland and abroad ${ }^{[1-4}$.

To solve one-dimension parameter estimation for distributed sources, [5 introduces angle expansion into the representation of sources and presents a parameter estimation algorithm for distributed sources (DSPE), which needs two-dimension search and bases on the assumption that all sources have the same angular signal density. [6 gives a TLS-ESPRIT algorithm using Taylor series approximation of array steering vectors. This algorithm reduces the computation complexity while it relies on special array structure. [7 introduces a covariance fitting method which is suitable for uncorrelated distributed sources. Although this algorithm dose not need high dimension searching and has low complexity, it dose need the original information of sources' DOA.

For the two-dimension parameters estimation for distributed sources, [8 realized two-dimension DOA estimation using peak-searching methods, which need fourdimension searching and increased computation complexity. Algorithm in [9 reduced complexity by converting twodimension parameters estimation to one-dimension parameters estimation. However, two uniform circular array are needed which increases the difficulty of hardware realization.

\section{SignAL MODEL}

Array structure in this paper is as figure 1

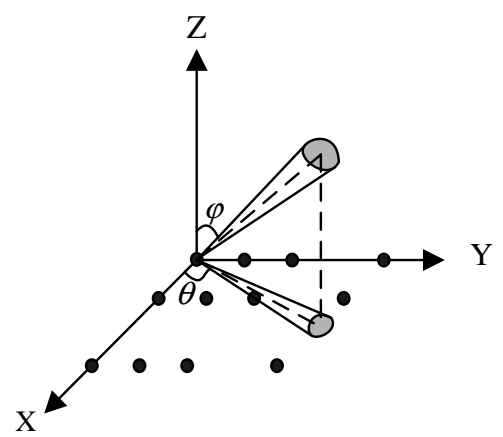

Fig.1 rectangle array structure

Several narrowband coherent distributed sources impinge on a $M_{1} \times M_{2}$ uniform rectangle array. Distance between adjacent array-element is $d$. Observed data vectors can be expressed as

$$
\boldsymbol{X}(t)=\iint \boldsymbol{a}(\vartheta, \phi) s(t) d \vartheta d \phi+\boldsymbol{n}(t)
$$

Where $\boldsymbol{a}(\vartheta, \phi)$ is steering vector of the point source, $s(t)$ is complex envelop of the signal, and $\boldsymbol{n}(t)$ is additive white Gaussian noise. The steering vector $\boldsymbol{a}(\vartheta, \phi)$ is defined by

$$
\begin{gathered}
\boldsymbol{a}(\vartheta, \phi)=\left[e^{-j 2 \pi(d / \lambda) \cos \varphi\left(p_{x_{1}} \sin \theta+p_{y_{2}} \cos \theta\right)},\right. \\
\left.\cdots, e^{-j 2 \pi(d / \lambda) \cos \varphi\left(p_{x_{M}} \sin \theta+p_{y_{M}} \cos \theta\right)}\right]^{\mathrm{T}}
\end{gathered}
$$

Where $\lambda$ is the wavelength, $p_{x_{m}}$ and $p_{y_{m}}$ are the coordinate of the $m$ th sensor in the antenna plane. 
Angular signal density of the distributed source is

$$
s(t)=\gamma \rho(\vartheta, \phi ; \boldsymbol{\mu})
$$

In equation (3), $\gamma$ is a random variable, $\rho(\vartheta, \phi ; \boldsymbol{\mu})$ is the deterministic angular signal density, $\boldsymbol{\mu}=\left(\theta, \sigma_{\theta}, \varphi, \sigma_{\varphi}\right)$ is the azimuth DOA, the angular spread of the azimuth DOA, the elevation DOA and the angular spread of the elevation DOA. Then steering vectors of coherent distributed sources are

$$
\boldsymbol{b}(\boldsymbol{\mu})=\iint \boldsymbol{a}(\vartheta, \phi) \rho(\vartheta, \phi ; \boldsymbol{\mu}) d \vartheta d \phi
$$

Gaussian distribution is the most familiar distribution type of distributed sources, whose $\rho(\vartheta, \phi ; \boldsymbol{\mu})$ is

$$
\rho(\vartheta, \phi ; \boldsymbol{\mu})=\frac{1}{2 \pi \sigma_{\theta} \sigma_{\varphi}} e^{-1 /\left((\vartheta-\theta)^{2} / \sigma_{\theta}^{2}+(\phi-\varphi)^{2} / \sigma_{\varphi}^{2}\right)}
$$

The received signal is given by

$$
\boldsymbol{X}(t)=s(t) \boldsymbol{b}(\boldsymbol{\mu})+\boldsymbol{n}(t)
$$

Covariance matrix of array observation signals is defined as

$$
\boldsymbol{R}=E\left\{\boldsymbol{X}(t) \boldsymbol{X}^{\mathrm{H}}(t)\right\}
$$

In paractical situations, the true covariance matrix of $\boldsymbol{X}(t)$ is unavailable but can be estimated. Therefore, the sample covariance matrix is defined as

$$
\hat{\boldsymbol{R}}=\frac{1}{N} \boldsymbol{X} \boldsymbol{X}^{\mathrm{H}}
$$

Where $N$ is the number of snapshot.

\section{TWO DIMENSION ESPRIT ALGORITHM FOR DISTRIBUTED SOURCES}

\section{A. Steering vector of coherent distributed source}

Steering vector of coherent distributed source under Gaussian distribution is

$$
\begin{gathered}
{[\boldsymbol{b}(\boldsymbol{\mu})]_{k}=\frac{1}{2 \pi \sigma_{\theta} \sigma_{\varphi}} \iint e^{j 2 \pi(d / \lambda) \cos \phi\left(p_{x_{m}} \sin \theta+p_{y_{m}} \cos \theta\right)}} \\
\times e^{-1 /\left((\vartheta-\theta)^{2} / \sigma_{\theta}^{2}+(\phi-\varphi)^{2} / \sigma_{\varphi}^{2}\right)} d \vartheta d \phi
\end{gathered}
$$

Where [ $]_{k}$ is the $k$ th element, $k=p_{x_{m}}+\left(p_{y_{m}}-1\right) M_{1}$, $1<p_{x_{1}}<M_{1}, 1<p_{x_{1}}<M_{2}$.

Set $\tilde{\theta}=\vartheta-\theta, \tilde{\phi}=\phi-\varphi$, the above equation is as below

$$
[\boldsymbol{b}(\boldsymbol{\mu})]_{k} \approx e^{j 2 \pi(d / \lambda) \cos \varphi\left(p_{x_{m}} \sin \theta+p_{y_{m}} \cos \theta\right)} \boldsymbol{g}_{k}(\boldsymbol{\mu})
$$

$$
\begin{aligned}
& {[\boldsymbol{g}(\boldsymbol{\mu})]_{k}=} \\
& \frac{1}{\sqrt{2 \pi} \sigma_{\theta}} \int e^{j 2 \pi(d / \lambda) \tilde{\vartheta}\left(p_{x_{m}} \sin \theta \cos \varphi-p_{y_{m}} \cos \theta \cos \varphi\right)} e^{-1 /\left(\tilde{\vartheta}^{2} / \sigma_{\theta}^{2}\right)} d \tilde{\vartheta} \\
& \frac{1}{\sqrt{2 \pi} \sigma_{\varphi}} \int e^{-j 2 \pi(d / \lambda) \tilde{\phi}\left(p_{x_{m}} \cos \theta \sin \varphi+p_{y_{m}} \sin \theta \sin \varphi\right)} e^{-1 /\left(\tilde{\phi}^{2} / \sigma_{\varphi}^{2}\right)} d \tilde{\phi}
\end{aligned}
$$

According to the integral equation listed below

$$
\begin{aligned}
& \int_{-\infty}^{\infty} e^{\left(-f^{2} x^{2}\right)} e^{[j p(x+\alpha)]} d x \\
& =\sqrt{\pi} e^{\left(-p^{2} /\left(4 f^{2}\right)\right)} \frac{e^{(j p \alpha)}}{f}
\end{aligned}
$$

Equation (11) is transformed into

$$
\begin{aligned}
& {[\boldsymbol{b}(\boldsymbol{\mu})]_{k} \approx e^{j 2 \pi(d / \lambda) \cos \varphi\left(p_{x_{m}} \sin \theta+p_{y_{m}} \cos \theta\right)}} \\
& \times e^{-2 \pi^{2}(d / \lambda)^{2} \sigma_{\theta}^{2}\left(p_{x_{m}} \sin \theta \cos \varphi+p_{y_{m}} \cos \theta \cos \varphi\right)^{2}} \\
& \times e^{-2 \pi^{2}(d / \lambda)^{2} \sigma_{\varphi}^{2}\left(-p_{x_{m}} \cos \theta \sin \varphi-p_{y_{m}} \sin \theta \sin \varphi\right)^{2}}
\end{aligned}
$$

The generalized steering vector $\boldsymbol{b}(\boldsymbol{\mu})$ is defined by

$$
\boldsymbol{b}\left(\theta, \sigma_{\theta}, \varphi, \sigma_{\varphi}\right)=\boldsymbol{a}(\theta, \varphi) \odot \boldsymbol{g}\left(\theta, \sigma_{\theta}, \varphi, \sigma_{\varphi}\right)
$$

Where $\odot$ is Schur-Hadamard.

For the uniform distribution, the $\boldsymbol{g}(\boldsymbol{\mu})$ is as below $[\boldsymbol{g}(\boldsymbol{\mu})]$

$=\frac{1}{2 \sigma_{\theta}} \int e^{j 2 \pi(d / \lambda) \tilde{\vartheta} \cos \varphi\left(p_{x_{m}} \cos \theta-p_{y_{m}} \sin \theta\right)} \operatorname{Rect}\left[-\sigma_{\theta}, \sigma_{\theta}\right] d \tilde{\vartheta}$

$\times \frac{1}{2 \sigma_{\varphi}} \int e^{-j 2 \pi(d / \lambda) \tilde{\phi} \sin \varphi\left(p_{x_{m}} \sin \theta+p_{y_{m}} \cos \theta\right)} \operatorname{Rect}\left[-\sigma_{\varphi}, \sigma_{\varphi}\right] d \tilde{\phi}(15)$

$=\sin c\left[2 \pi(d / \lambda) \sigma_{\theta} \cos \varphi\left(m_{1} \sin \theta-m_{2} \cos \theta\right)\right]$ $\times \sin c\left[2 \pi(d / \lambda) \sigma_{\varphi} \sin \varphi\left(\left(m_{1} \cos \theta+m_{2} \sin \theta\right)\right)\right]$

B. Azimuth estimation for coherent distributed sources Separate the array into four subarrays. $\left(1 \sim M_{1}-1\right) \times M_{2}$ array element along the X-axis consist of the first subarray, $\left(2 \sim M_{1}\right) \times M_{2}$ array element consist of the second subarray, $M_{1} \times\left(1 \sim M_{2}-1\right)$ array element along the y-axis consist of the third subarray, and $M_{1} \times\left(2 \sim M_{2}\right)$ array element consist of the fourth subarray.

Considering coherent distributed sources whose angular signal density meet Gaussian distribution, according to the(13), the generalized steering vector of respective subarray satisfies 


$$
\begin{aligned}
& \boldsymbol{b}_{2}(\boldsymbol{\mu})=\iint \boldsymbol{a}_{2}(\vartheta, \phi) \rho(\vartheta, \phi ; \boldsymbol{\mu}) d \vartheta d \phi \\
& =\iint \boldsymbol{a}_{1}(\vartheta, \phi) e^{j 2 \pi(d / \lambda) \cos \varphi \sin \theta} \rho(\vartheta, \phi ; \boldsymbol{\mu}) d \vartheta d \phi \\
& \approx \boldsymbol{\Phi}_{x} \boldsymbol{b}_{1}(\boldsymbol{\mu}) \\
& \boldsymbol{b}_{4}(\boldsymbol{\mu})=\iint \boldsymbol{a}_{3}(\vartheta, \phi) \rho(\vartheta, \phi ; \boldsymbol{\mu}) d \vartheta d \phi \\
& =\iint \boldsymbol{a}_{3}(\vartheta, \phi) e^{j 2 \pi(d / \lambda) \cos \varphi \cos \theta} \rho(\vartheta, \phi ; \boldsymbol{\mu}) d \vartheta d \phi \\
& \approx \boldsymbol{\Phi}_{y} \boldsymbol{b}_{3}(\boldsymbol{\mu})
\end{aligned}
$$

Where $\quad \boldsymbol{\Phi}_{x}=e^{j 2 \pi(d / \lambda) \cos \varphi \sin \theta} \cdot \operatorname{diag}\left[e^{u_{1}} \cdots e^{u_{\left(M_{1}-1\right) M_{2}}}\right]$, $\boldsymbol{\Phi}_{y}=e^{j 2 \pi(d / \lambda) \cos \varphi \cos \theta} \cdot \operatorname{diag}\left[e^{v_{1}} \cdots e^{v_{M_{1}(M 2-1)}}\right]$. Information of azimuth and elevation is all involved in the complex exponential elements of exponential items. That is to say, as long as we get the $\boldsymbol{\Phi}_{x}$ and $\boldsymbol{\Phi}_{y}$, DOA information of sources can be obtained for the phase information of the $\boldsymbol{\Phi}_{x}$ and $\Phi_{y}$.

Assume signal subspaces of these subarrays are $\boldsymbol{U}_{s 1}^{x}$, $\boldsymbol{U}_{s 2}^{x}, \quad \boldsymbol{U}_{s 1}^{y}, \quad \boldsymbol{U}_{s 2}^{y}$, respectively. Their relationship is as below

$$
\begin{aligned}
& \boldsymbol{U}_{s 2}^{x}=\boldsymbol{\Phi}_{x} \boldsymbol{U}_{s 1}^{x} \\
& \boldsymbol{U}_{s 2}^{y}=\boldsymbol{\Phi}_{y} \boldsymbol{U}_{s 1}^{y}
\end{aligned}
$$

That is

$$
\begin{aligned}
& \boldsymbol{\Phi}_{x}=\boldsymbol{U}_{s 2}^{x}\left(\boldsymbol{U}_{s 1}^{x}\right)^{+} \\
& \boldsymbol{\Phi}_{y}=\boldsymbol{U}_{s 2}^{y}\left(\boldsymbol{U}_{s 1}^{y}\right)^{+}
\end{aligned}
$$

Where + denotes pseudo-inverse.

As there is only one distributed source, information of DOA is involved in the largest eigenvalue of equation (20) and (21). After eigen-decomposition for $\boldsymbol{\Phi}_{x}, \boldsymbol{\Phi}_{y}$, the largest eigenvalues of equation (20) and (21) are acquired, which are $\gamma_{x}$ and $\gamma_{y}$. Then

$$
\begin{gathered}
\hat{\theta}=\sin ^{-1}\left\{\frac{\lambda}{2 \pi d}\left[\arg ^{2}\left(\gamma_{x}\right)+\arg ^{2}\left(\gamma_{y}\right)\right]^{1 / 2}\right\} \\
\hat{\varphi}=\tan ^{-1}\left[\frac{\arg \left(\gamma_{x}\right)}{\arg \left(\gamma_{y}\right)}\right]
\end{gathered}
$$

Although the above conclusion is achieved under the assumption of Gaussian sources, it is not restricted with angular signal density. So, the above conclusion is the same with any other sources with arbitrary angular signal density.

\section{Simulation}

In this section, we investigate the performance of the proposed algorithm through some simulation experiments. Assume that the uniform rectangle array has
$M=4 \times 4$ sensors, whose array element space is $d=0.5 \lambda$, the signal-to-noise ratio(SNR) is defined as $10 \log \sigma^{2}$. In the first example, we numerically illustrate the estimation performance of the proposed algorithm for coherent distributed source when the SNR varies, the nominal DOA of the distributed source is $\left(10^{\circ}, 30^{\circ}\right)$, angle pervasion is $\left(5^{\circ}, 3^{\circ}\right)$, snapshot is 1024 , the number of MonteCarlo experiment is 200 , and SNR varies between $-10 \mathrm{~dB}$ and 30dB.

According to Fig.2, RMSE for azimuth and elevation of present method gradually reduce along with the reduction of SNR. Under low SNR, RMSE for azimuth and elevation are all within $1^{\circ}$. Present method is suitable for both Gaussian distributed source as well as uniform distributed source, and RMSE under Gaussian distribution is a little larger than that under uniform distribution.

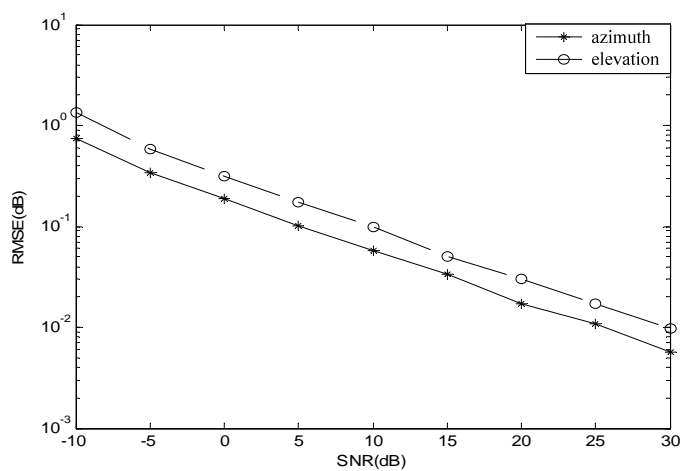

a) Gaussian distribution

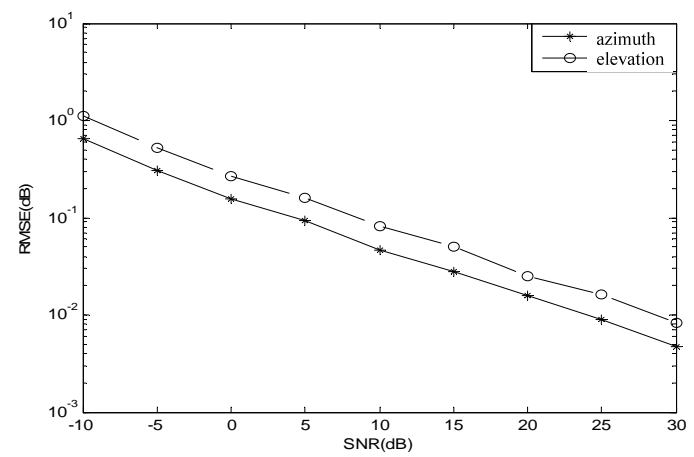

b) Uniform distribution

Fig.2 The RMSE of the nominal DOA versus SNR for a distributed source.

In the third example, we examine the estimation performance of the proposed algorithm for a Gaussianshaped distributed source when the angular spread $\sigma_{\theta}$ and $\sigma_{\varphi}$ varies. Set SNR as $10 \mathrm{~dB}$, angle pervasion for azimuth and elevation of distributed source is between $0^{\circ} \sim 10^{\circ}$, and other conditions are the same as the above simulation. 


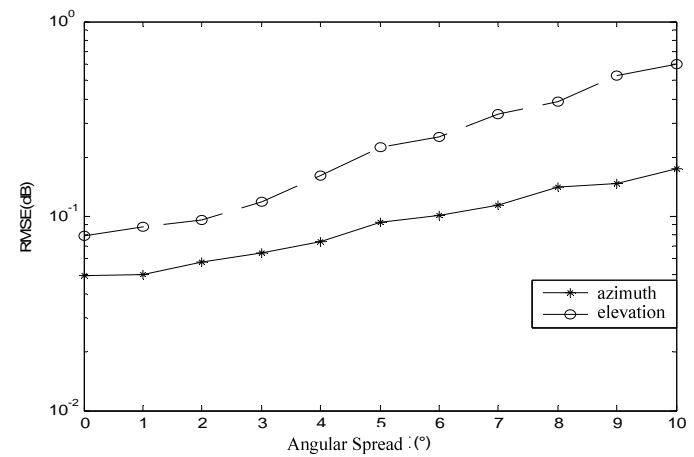

a) Gaussian distribution

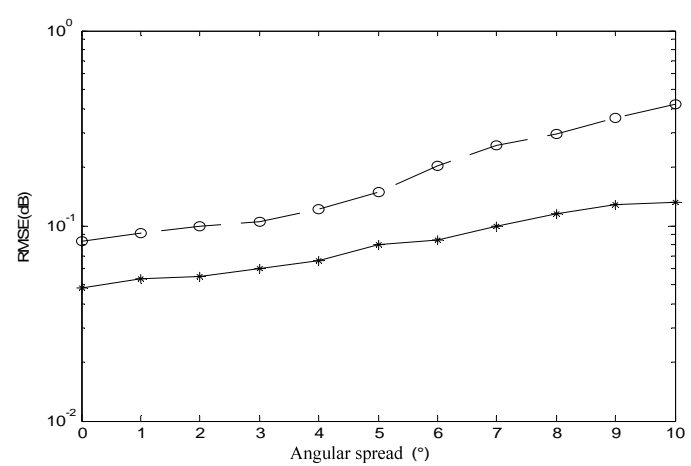

b) Uniform distribution

Fig.3 The RMSE of the nominal DOA versus angular spread for a distributed source.

In Fig.3, when angle pervasion is large, RMSE performance of introduced method degrades. However, it still effectively obtains the DOAs of impinging source. Moreover, influence of angle pervasion under guassian distribution is larger than that under uniform distribution.

\section{CONCLUSIONS}

Utilizing rotational invariance property of signal subspace of distributed sources, an ESPRIT algorithm of DOA estimation for coherent distributed sources is introduced. The method does not need peak searching, reduces the computational complexity, is suitable for sources with large pervasion while angular signal density satisfies Gaussian distribution and uniform distribution, and is not impacted with the angular signal density distribution of sources. Simulation implies that this algorithm can provide fine estimation precision

\section{REFERENCES}

[1] D. Asztely, B. Ottersten, and A. L. Swindlehurst, "Generalized array manifold for wireless communication channels with local scattering,” IEEE processing-F, vol.145, No.1, pp. 51-57, 1995.

[2] M. Bengtsson, B. Ottersten , "Low-complexity estimators for distributed sources”, IEEE Trans.Signal Processing, vol.48, pp.21852194, 2000.

[3] S. Hassanien, S. Shahbazpanahi, and A. B. Gershman, "A generalized Capon estimator for localization of multiple spread sources," IEEE Trans. on Signal Processing, vol.52, No.1, pp.280283, 2004.
[4] J. Lee, J. Joung, J. D. Kim, “A method for the direction-of-arrival estimation of inchoherently distributed sources”, IEEE Trans. on Vehicular Technology, vol.57, pp.2885-2893, May 2008.

[5] S. Valaee, B. Champagne, and P. Kabal, "Parametric localization of distributed sources," IEEE Trans.Signal Processing, vol.43, No.9, pp.2144-2153, 1995.

[6] S. Shahbazpanahi, M. H. Bastani, "Distributed source localization using ESPRIT algorithm,” IEEE Trans. on Signal Processing, vol.49, No.10, pp-2169-2178, 2001.

[7] A. Zoubir, Y. Wang, P. Charge, "Efficient subspace-based estimator for localization of multiple incoherently distributed sources,” IEEE Trans.Signal Processing, vol.56, No.2, pp.532-542, 2008.

[8] Q. Wan, Y. N. Peng, "Low-complexity estimator for fourdimensional parameters under a reparameterised distributed source model,” IEE Proc.-Radar. Sonar Navig., vol. 148, No.6, pp. 313$317,2001$.

[9] J. Lee, L. Song, H. Kwon, and S. R. Lee, "Low-complexity estimation of 2D DOA for coherently distributed sources," Signal Processing, vol.83, pp.1789-1802,2003. 